\title{
Error analysis of exponential integrators for oscillatory second-order differential equations
}

\author{
Volker Grimm, Marlis Hochbruck \\ Mathematisches Institut, Heinrich-Heine Universität Düsseldorf, Universitätsstraße 1, \\ D-40225 Düsseldorf, Germany \\ E-mail: \{grimm,marlis\}@am.uni-duesseldorf.de
}

\begin{abstract}
In this paper we analyse a family of exponential integrators for secondorder differential equations in which high-frequency oscillations in the solution are generated by a linear part. Conditions are given which guarantee that the integrators allow second-order error bounds independent of the product of the step size with the frequencies. Our convergence analysis generalises known results on the mollified impulse method by García-Archilla, Sanz-Serna and Skeel [6] and on Gautschi-type exponential integrators $[12,13]$.
\end{abstract}

AMS classification scheme numbers: 65L05, 65L70, 65M12

Keywords: Exponential integrators, oscillatory differential equation, error bounds

Submitted to: Journal of Physics A, Special Issue-Geometric Integration, version of 6 January 2006 


\section{Introduction}

In this paper we present an error analysis for a family of exponential integrators for the solution of systems of second-order differential equations

$$
y^{\prime \prime}(t)=-A y(t)+g(y(t)), \quad y\left(t_{0}\right)=y_{0}, \quad y^{\prime}\left(t_{0}\right)=y_{0}^{\prime},
$$

where $A=\Omega^{2}$ is a positive semi-definite symmetric matrix of arbitrarily large norm. Such problems have been studied in a number of papers recently, see, e.g., $[1,2,3,4,6,8,9,10,11,12,13,14,15,16,17,18]$. So far, error bounds which do not deteriorate when the product of the step size with the frequencies of the problem become large or, in the case of resonances, close to multiples of $\pi$, have been proved for two different schemes. The first one is the mollified impulse method proposed and analysed by García-Archilla, Sanz-Serna and Skeel [6]. Using a different technique, Hochbruck and Lubich [13] considered a Gautschi-type exponential integrator and proved error bounds for a two-step formulation of the scheme. The analysis in [13] also gave new insight into the convergence of the mollified impulse method.

Recently, the implication of geometric properties like symplecticity, symmetry, or reversibility on the long-time behaviour of the schemes when applied to highly-oscillatory problems has been studied $[3,11,12]$. As a first attempt in understanding these phenomena, a model problem, which is a special case of (1),

$$
y^{\prime \prime}(t)=-\left[\begin{array}{cc}
0 & 0 \\
0 & \omega^{2} I
\end{array}\right] y(t)+g(y(t)), \quad y\left(t_{0}\right)=y_{0}, \quad y^{\prime}\left(t_{0}\right)=y_{0}^{\prime},
$$

with blocks of arbitrary dimension, was proposed. The behaviour of a whole family of exponential integrators, which includes the mollified impulse method [6] and the Gautschi-type integrator [13] as special cases, was analysed in detail for this model problem in [11]. The analysis showed that neither of the latter two methods is the best possible with respect to long-time behaviour. However, to the best of our knowledge, error bounds for the most promising methods for the general problem (1) are not known so far. Results based on the modulated Fourier expansion [3] have been proved for twostep methods for the model problem (2) only [12, Section XIII.4]. They can probably be generalised to the case that there is a finite number of large frequencies by using the techniques of [4].

In the present paper, we will characterise all possible methods of the family proposed in [12] which allow second-order error bounds for the general problem (1) by presenting a unified error analysis for the whole family of methods. The techniques used in [6] and [13] do not extend to this general class in an obvious way. In contrast to the analysis of [13], where the two-step version of the Gautschi-type method is considered, the present paper deals with the one-step formulation. A major advantage of our new analysis is that it does not require bounds for point-wise products of matrices and therefore, generalises to abstract differential equations, where $A$ is an unbounded operator with infinitely many large eigenvalues directly. A conjecture posed in [13] which states that the twostep formulation of the Gautschi-type methods allows for error bounds independent of 
the dimension of the problem was proved by Grimm in [9].

Our paper is organised as follows: we will recall the family of methods considered here in Section 2. The main theorem and a new choice of filter function is presented in Section 3. In order to compare the performance of our scheme to known results in the literature, in particular in [12, Chapter XIII], we have chosen to show the numerical behaviour of the methods by applying it to the Fermi-Pasta-Ulam problem. The results are presented in Section 4. It turns out that, for the Fermi-Pasta-Ulam problem, our new method is the only one which is of second order and conserves the energy for longtime intervals. Since the proof of our main theorem is quite involved it is postponed to Section 5 .

\section{The integration scheme}

The variation-of-constants formula

$$
\begin{aligned}
{\left[\begin{array}{c}
y(t) \\
y^{\prime}(t)
\end{array}\right]=} & {\left[\begin{array}{cc}
\cos \left(t-t_{0}\right) \Omega & \Omega^{-1} \sin \left(t-t_{0}\right) \Omega \\
-\Omega \sin \left(t-t_{0}\right) \Omega & \cos \left(t-t_{0}\right) \Omega
\end{array}\right]\left[\begin{array}{c}
y\left(t_{0}\right) \\
y^{\prime}\left(t_{0}\right)
\end{array}\right] } \\
& +\int_{t_{0}}^{t}\left[\begin{array}{c}
\Omega^{-1} \sin (t-s) \Omega \\
\cos (t-s) \Omega
\end{array}\right] g(y(s)) d s
\end{aligned}
$$

suggests the following numerical integration schemes for the solution of (1)

$$
\left[\begin{array}{l}
y_{n+1} \\
y_{n+1}^{\prime}
\end{array}\right]=R(h \Omega)\left[\begin{array}{l}
y_{n} \\
y_{n}^{\prime}
\end{array}\right]+\left[\begin{array}{c}
\frac{1}{2} h^{2} \Psi g\left(\Phi y_{n}\right) \\
\frac{1}{2} h\left(\Psi_{0} g\left(\Phi y_{n}\right)+\Psi_{1} g\left(\Phi y_{n+1}\right)\right)
\end{array}\right],
$$

where

$$
R(h \Omega):=\left[\begin{array}{cc}
\cos h \Omega & \Omega^{-1} \sin h \Omega \\
-\Omega \sin h \Omega & \cos h \Omega
\end{array}\right]
$$

and

$$
\Phi=\phi(h \Omega), \quad \Psi=\psi(h \Omega), \quad \Psi_{0}=\psi_{0}(h \Omega), \quad \Psi_{1}=\psi_{1}(h \Omega) .
$$

The functions $\phi, \psi, \psi_{0}$, and $\psi_{1}$ are even analytic functions, with

$$
\phi(0)=\psi(0)=\psi_{0}(0)=\psi_{1}(0)=1,
$$

bounded on the non-negative real axis. By exchanging $n \leftrightarrow n+1$ and $h \leftrightarrow-h$ in the method, it can be seen that the method is symmetric if and only if

$$
\psi(\xi)=\operatorname{sinc}(\xi) \psi_{1}(\xi), \quad \psi_{0}(\xi)=\cos (\xi) \psi_{1}(\xi),
$$

where $\operatorname{sinc} \xi=\sin \xi / \xi$. A symmetric method can be cast into an equivalent two-step formulation

$$
y_{n+1}-2 \cos h \Omega \cdot y_{n}+y_{n-1}=h^{2} \Psi g\left(\Phi y_{n}\right),
$$

with starting values

$$
y_{0}, \quad y_{1}=\cos h \Omega \cdot y_{0}+\Omega^{-1} \sin h \Omega \cdot y_{0}^{\prime}+\frac{1}{2} h^{2} \Psi g\left(\Phi y_{0}\right) .
$$


The methods are equivalent with these initial values only. Multistep methods are usually considered to be of second order if they are of second order for arbitrary starting values that are close enough to the exact solution. According to this definition, one-step methods (3) and their two-step formulation (6) are not equivalent with respect to their non-smooth order. For example, the mollified impulse method is of second order as a one-step method, but if one uses the exact solution as starting values $y_{0}$ and $y_{1}$, the mollified impulse method is not of second order as a two-step method, cf. [13, Section 7]. Another example is the Gautschi-type exponential integrator (6) in which

$$
\psi(\xi)=\operatorname{sinc}^{2}\left(\frac{1}{2} \xi\right), \quad \phi(\xi)=\operatorname{sinc}(\xi)\left(1+\frac{1}{3} \sin ^{2}\left(\frac{1}{2} \xi\right)\right) .
$$

This method is of non-smooth second order for arbitrary starting values close enough to the exact solution. However, if we rewrite this method in the one-step form (3), then the function $\psi$ satisfying the symmetry relation (5) yields a filter function $\psi_{1}$ that has singularities at odd integer multiples of $\pi$. This prevents the method from being of order two as a one-step method in the form (3). A different one-step formulation of the Gautschi-type method is given in [13]. Our paper focuses on one-step methods.

Symplectic methods possess a very good energy preservation for small step sizes $h$, whenever (1) is a Hamiltonian system. The methods are symplectic, if and only if

$$
\psi(\xi)=\operatorname{sinc}(\xi) \phi(\xi),
$$

cf. [12, p. 417]. However, with respect to oscillatory differential equations, where the product of the highest frequency in the system with the step size $h$ is large, the situation is different. The analysis in [11] for linear problems (2), i.e. $g(y)=B y$, satisfying the finite-energy condition

$$
\frac{1}{2}\left\|y^{\prime}(t)\right\|^{2}+\frac{1}{2}\|\Omega y(t)\|^{2} \leq \frac{1}{2} K^{2}, \quad t_{0} \leq t \leq t_{0}+T,
$$

shows that the numerical method conserves the total energy up to $O(h)$ for all values of $h \omega$, if and only if

$$
\psi(\xi)=\operatorname{sinc}^{2}(\xi) \phi(\xi),
$$

see [12, p. 449]. The relations (8) and (10) cannot be satisfied simultaneously, and symmetric methods that satisfy (10) are therefore expected to possess favourable energyconservation properties. It is not clear to us whether condition (10) is sufficient to guarantee energy preservation for general systems (1). But the analysis in [11] clearly shows that these methods are at least preferable to symplectic methods for oscillatory differential equations.

\section{Finite-time error analysis}

The result stated in this section makes no smoothness assumptions about the highlyoscillatory solution $y$ except (9). The even analytic functions defining the integrator (3) are assumed to be bounded on the non-negative real axis, i.e. $\chi=\phi, \psi, \psi_{0}, \psi_{1}$ satisfy

$$
\max _{\xi \geq 0}|\chi(\xi)| \leq M_{1}
$$


for some constant $M_{1}$. Moreover, we assume $\phi(0)=1$, thus the existence of a constant $M_{2}$ such that

$$
\max _{\xi \geq 0}\left|\frac{\phi(\xi)-1}{\xi}\right| \leq M_{2}
$$

In addition, we assume

$$
\max _{\xi \geq 0}\left|\frac{1}{\sin \frac{\xi}{2}}\left(\operatorname{sinc}^{2} \frac{\xi}{2}-\psi(\xi)\right)\right| \leq M_{3}
$$

and

$$
\max _{\xi \geq 0}\left|\frac{1}{\xi \sin \frac{\xi}{2}}(\operatorname{sinc} \xi-\chi(\xi))\right| \leq M_{4}, \quad \chi=\phi, \psi_{0}, \psi_{1} .
$$

The assumptions made so far are necessary to prove second-order error bounds for the positions $y_{n} \approx y\left(t_{n}\right)$. In order to verify first order error bounds for the velocities, we assume

$$
\max _{\xi \geq 0}|\xi \psi(\xi)| \leq M_{5}, \quad \max _{\xi \geq 0}\left|\frac{\xi}{\sin \frac{\xi}{2}}\left(\operatorname{sinc}^{2} \frac{\xi}{2}-\psi(\xi)\right)\right| \leq M_{6}
$$

and

$$
\max _{\xi \geq 0}\left|\frac{1}{\sin \frac{\xi}{2}}\left(\operatorname{sinc} \xi-\psi_{i}(\xi)\right)\right| \leq M_{7}, \quad i=0,1 .
$$

(16) is a consequence of (14), but possibly with $M_{7}>M_{4}$. The constants $M_{1}$ to $M_{7}$ only depend on the choice of the analytic functions. It is easy to find analytic functions such that

$$
M:=\max _{i=1, \ldots, 7} M_{i}
$$

is a small constant; examples will be given in Section 4.

Theorem 1. In (1), let $A=\Omega^{2}$ be an arbitrary symmetric positive semi-definite matrix. Suppose $g, g_{y}$ and $g_{y y}$ are bounded in the Euclidean norm or the norms induced by the Euclidean norm, respectively. Assume the solution y satisfies the finite-energy condition (9). If the even analytic functions of scheme (3) satisfy (11), (12), (13), and (14), then

$$
\left\|y\left(t_{n}\right)-y_{n}\right\| \leq h^{2} C, \quad t_{0} \leq t_{n}=t_{0}+n h \leq t_{0}+T .
$$

The constant $C$ only depends on $T, K, M_{1}, \ldots, M_{4},\|g\|,\left\|g_{y}\right\|$, and $\left\|g_{y y}\right\|$. If, in addition, (15) and (16) are satisfied, then

$$
\left\|y^{\prime}\left(t_{n}\right)-y_{n}^{\prime}\right\| \leq h \widetilde{C}, \quad t_{0} \leq t_{n}=t_{0}+n h \leq t_{0}+T .
$$

The constant $\widetilde{C}$ only depends on $T, K, M,\|g\|,\left\|g_{y}\right\|$, and $\left\|g_{y y}\right\|$.

The proof of this theorem will be given in Section 5 below.

It is important to note that the constants $C, \widetilde{C}$ only depend on the finite energy of the exact solution, the choice of the filter functions, and the smoothness of the nonlinearity $g$ but not on the norm of $A$ or on higher derivatives of the exact solution. This property is very desirable. For example, if system (1) is a semi-discretisation of a 
wave equation, then the bounds are independent of the mesh size used for the spatial discretisation.

If the method is symmetric (5) and symplectic $(8)$, with $\phi(\xi)$ vanishing at integer multiples of $\pi$, then (11)-(16) are satisfied. These are the assumptions stated in [6] and thus the results therein are a special case of our general Theorem 1.

However, it was shown in [11], that symmetric and symplectic methods cannot preserve the energy for linear systems (1) with $g(y)=B y$ for large step sizes. Interestingly, a symmetric method which additionally satisfies (10) instead of the symplecticity condition (8), with $\phi(\xi)$ vanishing at integer multiples of $\pi$, fulfils (11)(16). Therefore, the method with

$$
\phi(\xi)=\operatorname{sinc} \xi, \quad \psi(\xi)=\operatorname{sinc}^{2} \xi \phi(\xi),
$$

where $\psi_{0}$ and $\psi_{1}$ chosen such that the method is symmetric (5), fulfils all conditions of Theorem 1 and thus allows second-order error bounds independent of the frequencies. Moreover, it satisfies (10), so that long-time energy preservation similar to the method proposed in [11] can be expected. The latter method does not allow a second-order error bound independent of the norm of $\Omega$. This can be seen in Figure 2 in the plot labelled $(E)$, where the resonances appear exactly at the points where condition (14) for $\phi(\xi)=1$ fails to hold. A numerical comparison of the new method with existing schemes is given in the following section.

\section{Numerical Experiment}

We consider the Fermi-Pasta-Ulam problem, since this allows comparisons to earlier work, in particular in [12]. We refer the reader to [12] for a detailed description of this problem. To avoid confusion with the notation therein, we denote our new method with $(\mathrm{G})$. Since we only consider symmetric methods, it is enough to give the analytic functions $\psi$ and $\phi$ to determine the one-step method uniquely:
(A) $\psi(\xi)=\operatorname{sinc}^{2}\left(\frac{1}{2} \xi\right)$
$\phi(\xi)=1$
Gautschi [7]
(B) $\psi(\xi)=\operatorname{sinc}(\xi)$
$\phi(\xi)=1$
Deuflhard [5]
(C) $\psi(\xi)=\operatorname{sinc}(\xi) \phi(\xi)$
$\phi(\xi)=\operatorname{sinc}(\xi)$
García-Archilla et al. [6]
(D) $\psi(\xi)=\operatorname{sinc}^{2}\left(\frac{1}{2} \xi\right)$
$\phi(\xi)$ of (7)
Hochbruck, Lubich [13]
$(E) \quad \psi(\xi)=\operatorname{sinc}^{2}(\xi)$
$\phi(\xi)=1$
Hairer, Lubich [11]
(G) $\psi(\xi)=\operatorname{sinc}^{3}(\xi)$
$\phi(\xi)=\operatorname{sinc}(\xi)$

Figure 1 shows the maximum error of the total energy as a function of the scaled frequency $h \omega$ on the interval $[0,1000]$. Only methods $(E)$ and $(G)$ show a uniformly good energy preservation for all frequencies. To compare the accuracy, we used the Fermi-Pasta-Ulam problem with very stiff springs, $\omega=1000$. Methods $(C),(D)$, and $(G)$ are the only methods with uniformly good accuracy for all frequencies, as can be seen in Figure 2. Method $(G)$ is the only method that has a good behaviour with respect to accuracy and energy conservation uniformly in the frequencies for the FermiPasta-Ulam problem. Theorem 1 and the result about the conservation of energy for 
linear problems in [11] suggested this new method. The Fermi-Pasta-Ulam problem has another nearly conserved quantity, the oscillatory energy

$$
I=I_{1}+I_{2}+I_{3} \quad \text { with } \quad I_{j}=\frac{1}{2}\left(y^{\prime}\right)_{2, j}^{2}+\frac{1}{2} \omega^{2} y_{2, j}^{2} .
$$

Figure 3 shows the maximum error of the oscillatory energy. It can be seen that method $(G)$ is the only method that has a uniformly good preservation of the oscillatory energy for $h \omega$ bounded away from zero. This good performance compared to the other methods comes as a surprise. In [11], Hairer and Lubich could show that no method can uniformly conserve the oscillatory energy in an interval of length more than $2 \pi$ for linear systems. Methods (A)-(F) of [11] show severe resonances for the oscillatory energy in any interval of length more than $2 \pi$ in the nonlinear Fermi-Pasta-Ulam problem. The new method $(\mathrm{G})$ does not show severe resonances in the oscillatory energy for the Fermi-Pasta-Ulam Problem even on a finer temporal grid than that shown in the figure.
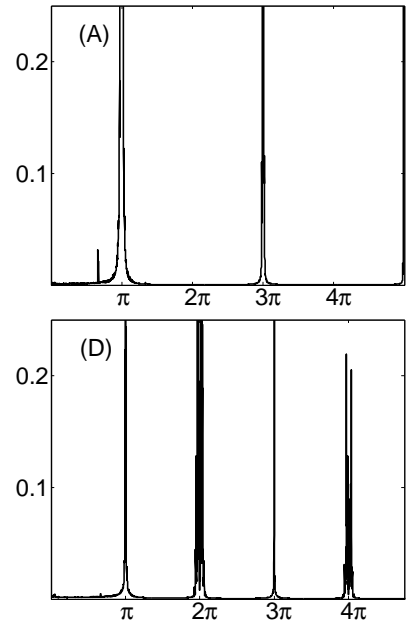
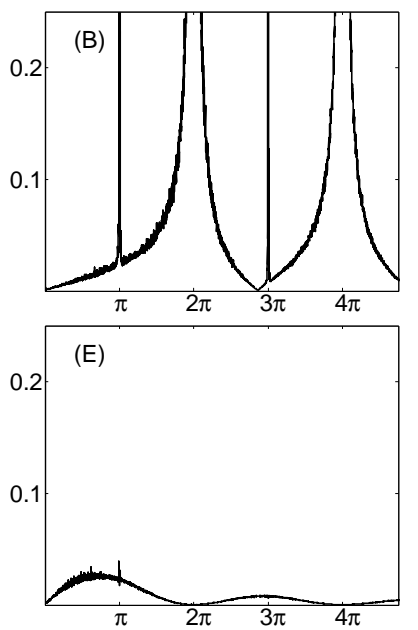

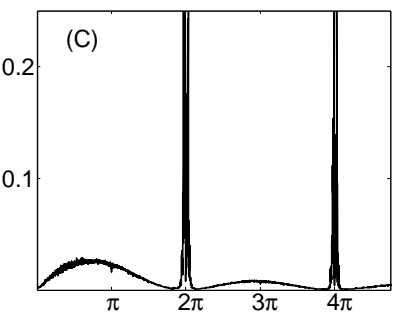

$(\mathrm{G})$

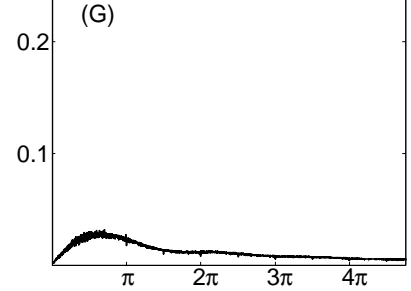

Figure 1. Maximum error of total energy on the interval $[0,1000]$ as a function of $h \omega$ (step size $h=0.02)$.

\section{Proof of Theorem 1}

The proof of Theorem 1 is tedious and split into several lemmas. In the following, $C$ is a generic constant, depending only on the constants mentioned in Theorem 1 , that takes on different values on different occurrences. By assumption, $\Omega=A^{1 / 2}$ is symmetric, positive semi-definite. If $\Omega$ is singular, $\Omega^{-1} \sin t \Omega$ is interpreted as $t \operatorname{sinc} t \Omega$, which is defined for an arbitrary matrix $\Omega$.

Proof of Theorem 1. Substitution of the exact solution into the integration scheme (3) with $R=R(h \Omega)$ gives

$$
\left[\begin{array}{c}
y\left(t_{n+1}\right) \\
y^{\prime}\left(t_{n+1}\right)
\end{array}\right]=R\left[\begin{array}{c}
y\left(t_{n}\right) \\
y^{\prime}\left(t_{n}\right)
\end{array}\right]+\left[\begin{array}{c}
\frac{1}{2} h^{2} \Psi g\left(\Phi y\left(t_{n}\right)\right) \\
\frac{1}{2} h\left(\Psi_{0} g\left(\Phi y\left(t_{n}\right)\right)+\Psi_{1} g\left(\Phi y\left(t_{n+1}\right)\right)\right)
\end{array}\right]+\left[\begin{array}{c}
d_{n} \\
d_{n}^{\prime}
\end{array}\right],
$$



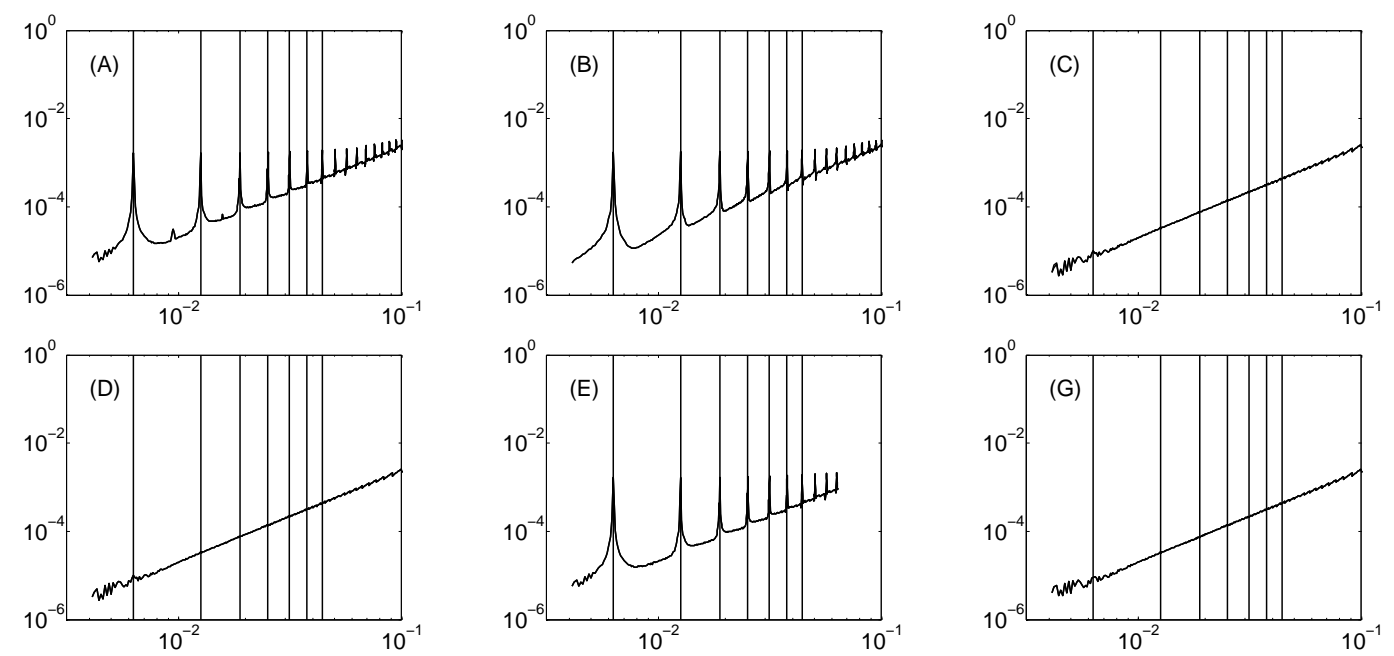

Figure 2. Global error in positions at $t=1$ of the methods versus step size
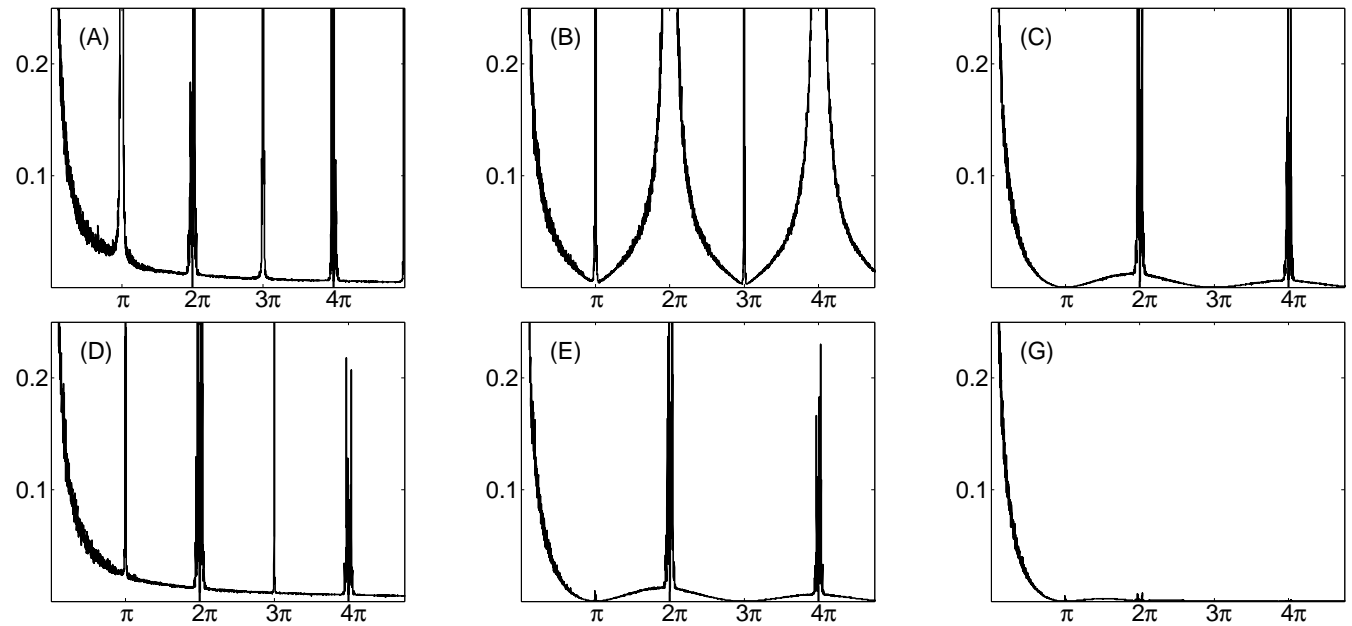

Figure 3. Maximum deviation of the oscillatory energy on the interval $[0,1000]$ as a function of $h \omega$ (step size $h=0.02$ ).

with the defects $d_{n}$ and $d_{n}^{\prime}$. Subtraction of equation (3) and summation leads to

$$
\left[\begin{array}{l}
e_{n+1} \\
e_{n+1}^{\prime}
\end{array}\right]=R^{n+1}\left[\begin{array}{c}
e_{0} \\
e_{0}^{\prime}
\end{array}\right]+\sum_{j=0}^{n} R^{n-j}\left[\begin{array}{c}
\frac{1}{2} h^{2} \Psi F_{j} e_{j} \\
\frac{1}{2} h \Psi_{0} F_{j} e_{j}+\frac{1}{2} h \Psi_{1} F_{j+1} e_{j+1}
\end{array}\right]+\left[\begin{array}{c}
D_{n} \\
D_{n}^{\prime}
\end{array}\right],
$$

where $e_{n}:=y\left(t_{n}\right)-y_{n}$ and $e_{n}^{\prime}:=y^{\prime}\left(t_{n}\right)-y_{n}^{\prime}$,

$$
F_{n}:=\int_{0}^{1} g_{y}\left(\Phi\left(y_{n}+u e_{n}\right)\right) d u \cdot \Phi, \quad\left\|F_{n}\right\| \leq\left\|g_{y}\right\| M_{1},
$$

and

$$
\left[\begin{array}{l}
D_{n} \\
D_{n}^{\prime}
\end{array}\right]=\sum_{j=0}^{n}\left[\begin{array}{cc}
\cos (n-j) h \Omega & \Omega^{-1} \sin (n-j) h \Omega \\
-\Omega \sin (n-j) h \Omega & \cos (n-j) h \Omega
\end{array}\right]\left[\begin{array}{l}
d_{j} \\
d_{j}^{\prime}
\end{array}\right]
$$


The proof proceeds as follows: we start by giving expressions for $d_{j}$ and $d_{j}^{\prime}$ in Lemma 1 and Lemma 2, respectively. Using these expressions, we provide bounds for the four sums in the right-hand side of (19) in Lemmas 3-6. These bounds yield

$$
\left\|D_{n}\right\| \leq C h^{2} \quad \text { and } \quad\left\|D_{n}^{\prime}\right\| \leq C h .
$$

Due to $e_{0}=e_{0}^{\prime}=0$ the recursion (18) reads

$$
e_{n+1}=h \sum_{j=1}^{n} L_{j} e_{j}+D_{n},
$$

where

$$
\begin{aligned}
L_{j}:=\frac{1}{2}(h \cos (n-j) h \Omega \cdot \Psi & +(n-j) h \operatorname{sinc}(n-j) h \Omega \cdot \Psi_{0} \\
& \left.\left.+(n+1-j) h \operatorname{sinc}(n+1-j) h \Omega \cdot \Psi_{1}\right)\right) F_{j} .
\end{aligned}
$$

This yields

$$
\left\|L_{j}\right\| \leq \frac{3}{2} T\left\|g_{y}\right\| M_{1}^{2}
$$

so that $\left\|e_{n}\right\| \leq C h^{2}$ follows from Gronwall's Lemma. Assumption (15) and the recursion for $e_{n}^{\prime}$ finally shows $\left\|e_{n}^{\prime}\right\| \leq C h$.

Lemma 1. The defects $d_{n}$ can be written as

$$
d_{n}=\frac{1}{2} h^{2}\left(\operatorname{sinc}^{2} \frac{h}{2} \Omega-\Psi\right) g\left(\Phi y\left(t_{n}\right)\right)+h^{3} z_{n}
$$

with

$$
\left\|z_{n}\right\| \leq C \quad \text { and } \quad\left\|h \Omega z_{n}\right\| \leq C .
$$

Thereby $C$ only depends on $K,\left\|g_{y}\right\|$, and $M_{2}$.

Proof. With the help of the variation-of-constants formula, the defects $d_{n}$ are given as

$$
d_{n}=\int_{t_{n}}^{t_{n+1}} \Omega^{-1} \sin \left(\left(t_{n+1}-s\right) \Omega\right) g(y(s)) d s-\frac{1}{2} h^{2} \Psi g\left(\Phi y\left(t_{n}\right)\right) .
$$

Transforming the integration interval to $[0,1]$, applying the variation-of-constants formula and Taylor expansion for $g$ leads to the representation given above with

$$
\begin{aligned}
& z_{n}=\int_{0}^{1}(1-s) \operatorname{sinc} h(1-s) \Omega \cdot( \\
& \int_{0}^{1} g_{y}\left(y\left(t_{n}\right)+u\left(y\left(t_{n}+h s\right)-y\left(t_{n}\right)\right)\right) d u\left[s \int_{0}^{1} y^{\prime}\left(t_{n}+h s v\right) d v\right] \\
&\left.-\int_{0}^{1} g_{y}\left(y\left(t_{n}\right)+u(\Phi-I) y\left(t_{n}\right)\right) d u\left[\frac{\Phi-I}{h \Omega} \Omega y\left(t_{n}\right)\right]\right) d s .
\end{aligned}
$$

Hence we have

$$
\left\|z_{n}\right\| \leq \frac{1}{2}\left(\frac{1}{3}+M_{2}\right)\left\|g_{y}\right\| K
$$

The bound for $\left\|h \Omega z_{n}\right\|$ follows by multiplying the equation above with $h \Omega$ since $(1-s)$ drops out and sinc turns into sin. 
Lemma 2. The defects $d_{n}^{\prime}$ can be written as

$$
\begin{aligned}
& d_{n}^{\prime}= \frac{1}{2} h\left(\operatorname{sinc} h \Omega-\Psi_{0}\right) g\left(\Phi y\left(t_{n}\right)\right)+\frac{1}{2} h\left(\operatorname{sinc} h \Omega-\Psi_{1}\right) g\left(\Phi y\left(t_{n}+h\right)\right) \\
&+ \frac{1}{2} h \int_{0}^{1} \cos h(1-s) \Omega \cdot g_{y}\left(\Phi y\left(t_{n}\right)\right) \cdot \\
& {\left[(\cos h s \Omega-\Phi) y\left(t_{n}\right)+\Omega^{-1} \sin h s \Omega \cdot y^{\prime}\left(t_{n}\right)\right] d s } \\
&+ \frac{1}{2} h \int_{0}^{1} \cos h(1-s) \Omega \cdot g_{y}\left(\Phi y\left(t_{n+1}\right)\right) \cdot \\
& {\left[(\cos h(s-1) \Omega-\Phi) y\left(t_{n}+h\right)\right.} \\
&\left.+\Omega^{-1} \sin h(s-1) \Omega \cdot y^{\prime}\left(t_{n}+h\right)\right] d s+h^{3} z_{n}^{\prime}
\end{aligned}
$$

with $\left\|z_{n}^{\prime}\right\| \leq C$. Here $C$ only depends on $K,\|g\|,\left\|g_{y}\right\|,\left\|g_{y y}\right\|$, and $M_{2}$.

Proof. The defects are given as

$$
\begin{aligned}
d_{n}^{\prime}= & \int_{0}^{h} \cos (h-s) \Omega \cdot g\left(y\left(t_{n}+s\right)\right) d s-\frac{1}{2} h\left(\Psi_{0} g\left(\Phi y\left(t_{n}\right)\right)+\Psi_{1} g\left(\Phi y\left(t_{n+1}\right)\right)\right) \\
= & \frac{1}{2} h\left(\operatorname{sinc} h \Omega-\Psi_{0}\right) g\left(\Phi y\left(t_{n}\right)\right)+\frac{1}{2} h\left(\operatorname{sinc} h \Omega-\Psi_{1}\right) g\left(\Phi y\left(t_{n}+h\right)\right) \\
& +\frac{1}{2} h \int_{0}^{1} \cos h(1-s) \Omega \cdot\left(g\left(y\left(t_{n}+h s\right)\right)-g\left(\Phi y\left(t_{n}\right)\right)\right) d s \\
& +\frac{1}{2} h \int_{0}^{1} \cos h(1-s) \Omega \cdot\left(g\left(y\left(t_{n}+h s\right)\right)-g\left(\Phi y\left(t_{n}+h\right)\right)\right) d s .
\end{aligned}
$$

Taylor expansion for $g$ and the variation-of-constants formula lead to the representation given above with $\left\|z_{n}^{\prime}\right\| \leq \frac{1}{6}\|g\|\left\|g_{y}\right\|+\left\|g_{y y}\right\|\left(1+M_{2}\right)^{2} K^{2}$.

Lemma 3. For $n$ with $0 \leq(n+1) h \leq T$, it holds that

$$
\left\|\sum_{j=0}^{n} \cos (n-j) h \Omega \cdot d_{j}\right\| \leq C h^{2}
$$

where $C$ depends on $K, T,\|g\|,\left\|g_{y}\right\|, M_{1}, M_{2}$, and $M_{3}$.

Proof. According to Lemma 1, we have

$$
\left\|h^{3} \sum_{j=0}^{n} \cos (j h \Omega) \cdot z_{n-j}\right\| \leq T C h^{2},
$$

where $C$ depends on $K,\left\|g_{y}\right\|$ and $M_{2}$. Thus it remains to bound

$$
\frac{1}{2} h^{2} \sum_{j=0}^{n} \cos (j h \Omega)\left(\operatorname{sinc}^{2} \frac{h}{2} \Omega-\Psi\right) g_{n-j}=: \frac{1}{2} h^{2} u_{n}, \quad g_{j}=g\left(\Phi y\left(t_{j}\right)\right) .
$$


By partial summation, $u_{n}$ can be written as

$$
u_{n}=E_{n}(h \Omega) g_{0}+\sum_{j=0}^{n-1} E_{j}(h \Omega)\left(g_{n-j}-g_{n-j-1}\right),
$$

where

$$
E_{j}(\xi):=\frac{1}{2 \sin \left(\frac{\xi}{2}\right)}\left(\operatorname{sinc}^{2} \frac{\xi}{2}-\psi(\xi)\right)\left(\sin \left(j \xi+\frac{\xi}{2}\right)+\sin \frac{\xi}{2}\right) .
$$

Due to (13), we have $\left\|E_{j}(h \Omega)\right\| \leq M_{3}$ and therefore

$$
\left\|u_{n}\right\| \leq M_{3}\|g\|+\sum_{j=0}^{n-1} M_{3} M_{1}\left\|g_{y}\right\| h K \leq M_{3}\left(\|g\|+T M_{1}\left\|g_{y}\right\| K\right) .
$$

This completes the proof.

Lemma 4. For $n$ with $0 \leq(n+1) h \leq T$, it holds that

$$
\left\|\sum_{j=0}^{n} \Omega^{-1} \sin (n-j) h \Omega \cdot d_{j}^{\prime}\right\| \leq C h^{2}
$$

where $C$ depends on $T, K,\|g\|,\left\|g_{y}\right\|,\left\|g_{y y}\right\|, M_{1}, M_{2}$, and $M_{4}$.

Proof. According to Lemma 2, it remains to bound

$$
\begin{aligned}
& \sum_{j=0}^{n} \Omega^{-1} \sin (n-j) h \Omega \cdot\left\{\frac{1}{2} h\left(\operatorname{sinc} h \Omega-\Psi_{0}\right) g\left(\Phi y\left(t_{j}\right)\right)\right. \\
& +\frac{1}{2} h\left(\operatorname{sinc} h \Omega-\Psi_{1}\right) g\left(\Phi y\left(t_{j}+h\right)\right) \\
& +\frac{1}{2} h \int_{0}^{1} \cos h(1-s) \Omega \cdot g_{y}\left(\Phi y\left(t_{j}\right)\right) \cdot \\
& \quad\left[(\cos h s \Omega-\Phi) y\left(t_{j}\right)+\Omega^{-1} \sin h s \Omega \cdot y^{\prime}\left(t_{j}\right)\right] d s \\
& +\frac{1}{2} h \int_{0}^{1} \cos h(1-s) \Omega \cdot g_{y}\left(\Phi y\left(t_{j+1}\right)\right) \cdot \\
& \quad\left[(\cos h(s-1) \Omega-\Phi) y\left(t_{j}+h\right)\right. \\
& \left.\left.+\Omega^{-1} \sin h(s-1) \Omega \cdot y^{\prime}\left(t_{j}+h\right)\right] d s\right\}
\end{aligned}
$$

The first and the second sum within the curly braces, (20) and (21), can be seen to be bounded by partial summation as in the lemma above. The third and the fourth term, (22) and (23), require more work. The third term (22) can be written as

$$
a_{n}=a_{n}^{(1)}+a_{n}^{(2)},
$$

where $G_{j}=g_{y}\left(\Phi y\left(t_{j}\right)\right)$ and

$$
\begin{aligned}
a_{n}^{(1)}=\frac{1}{2} h^{3} \sum_{j=0}^{n} \sin (n-j) h \Omega \cdot \int_{0}^{1}(1-s)\left(\frac{\cos h(1-s) \Omega-I}{h(1-s) \Omega}\right) G_{j} . \\
\\
{\left[s \frac{\cos h s \Omega-I}{h s \Omega} \Omega y\left(t_{j}\right)+\frac{I-\phi(h \Omega)}{h \Omega} \Omega y\left(t_{j}\right)+s \operatorname{sinc} h s \Omega \cdot y^{\prime}\left(t_{j}\right)\right] d s, }
\end{aligned}
$$


and

$$
a_{n}^{(2)}=\frac{1}{2} h \sum_{j=0}^{n} \Omega^{-1} \sin (n-j) h \Omega \cdot \int_{0}^{1} G_{j}\left[(\cos h s \Omega-\Phi) y\left(t_{j}\right)+\Omega^{-1} \sin h s \Omega \cdot y^{\prime}\left(t_{j}\right)\right] d s .
$$

Here, we wrote $1 / \Omega$ instead of $\Omega^{-1}$ in order to improve the readability. We have

$$
\left\|a_{n}^{(1)}\right\| \leq \frac{1}{2} h^{2} T\left(\frac{1}{3}+M_{2}\right)\left\|g_{y}\right\| K .
$$

Analogously, the fourth term, (23), can be written as

$$
b_{n}=b_{n}^{(1)}+b_{n}^{(2)} \text {, }
$$

where

$$
\begin{gathered}
b_{n}^{(1)}=\frac{1}{2} h^{3} \sum_{j=0}^{n} \sin (n-j) h \Omega \cdot \int_{0}^{1}(1-s)\left(\frac{\cosh (1-s) \Omega-I}{h(1-s) \Omega}\right) G_{j+1} . \\
{\left[(s-1) \frac{\cos h(s-1) \Omega-I}{h(s-1) \Omega} \Omega y\left(t_{j+1}\right)+\frac{I-\phi(h \Omega)}{h \Omega} \Omega y\left(t_{j+1}\right)\right.} \\
\left.+(1-s) \operatorname{sinc} h s \Omega \cdot y^{\prime}\left(t_{j+1}\right)\right] d s
\end{gathered}
$$

and

$$
\begin{aligned}
b_{n}^{(2)}=\frac{1}{2} h & \sum_{j=0}^{n} \Omega^{-1} \sin (n-j) h \Omega . \\
& \int_{0}^{1} G_{j+1}\left[(\cos h(s-1) \Omega-\Phi) y\left(t_{j+1}\right)+\Omega^{-1} \sin h(s-1) \Omega \cdot y^{\prime}\left(t_{j+1}\right)\right] d s .
\end{aligned}
$$

One readily observes

$$
\left\|b_{n}^{(1)}\right\| \leq \frac{1}{2} h^{2} T\left(\frac{2}{3}+M_{2}\right)\left\|g_{y}\right\| K .
$$

Hence it remains to bound $a_{n}^{(2)}+b_{n}^{(2)}$. Using

$$
\int_{0}^{1} \cos h s \Omega d s=\int_{0}^{1} \cos h(s-1) \Omega d s=\operatorname{sinc} h \Omega
$$

and

$$
\int_{0}^{1} \Omega^{-1} \sin h s \Omega d s=-\int_{0}^{1} \Omega^{-1} \sin h(s-1) \Omega d s=\frac{h}{2} \operatorname{sinc}^{2} \frac{h}{2} \Omega,
$$

one can rewrite

$$
\begin{aligned}
a_{n}^{(2)}+b_{n}^{(2)}= & \frac{1}{2} h \sum_{j=0}^{n} W_{n-j} G_{j}(\operatorname{sinc} h \Omega-\Phi) y\left(t_{j}\right) \\
& +\frac{1}{2} h \sum_{j=0}^{n} W_{n-j} G_{j+1}(\operatorname{sinc} h \Omega-\Phi) y\left(t_{j+1}\right) \\
& +\frac{h^{2}}{4} \sum_{j=0}^{n} W_{n-j} G_{j} \operatorname{sinc}^{2} \frac{h}{2} \Omega \cdot y^{\prime}\left(t_{j}\right) \\
& -\frac{h^{2}}{4} \sum_{j=0}^{n} W_{n-j} G_{j+1} \operatorname{sinc}^{2} \frac{h}{2} \Omega \cdot y^{\prime}\left(t_{j+1}\right),
\end{aligned}
$$


where

$$
W_{j}:=\Omega^{-1} \sin j h \Omega .
$$

Since (26)-(27) can be written as

$$
\frac{h^{2}}{4} W_{n} G_{0} \operatorname{sinc}^{2} \frac{h}{2} \Omega \cdot y^{\prime}\left(t_{0}\right)+\frac{h^{2}}{4} \sum_{j=1}^{n}\left(W_{n-j}-W_{n+1-j}\right) G_{j} \operatorname{sinc}^{2} \frac{h}{2} \Omega \cdot y^{\prime}\left(t_{j}\right)
$$

and

$$
\left\|W_{n-j}-W_{n+1-j}\right\|=\left\|h \int_{0}^{1} \cos h(n-j+s) \Omega d s\right\| \leq h \quad \text { and } \quad\left\|W_{j}\right\| \leq T,
$$

the sums in (26) and (27) are bounded. After once more applying the variation-ofconstants formula to $y\left(t_{j}\right)$ in $(24)$ and using a trigonometric identity, one has to bound

$$
\begin{aligned}
& \frac{h^{2}}{2} \sum_{j=0}^{n} W_{n-j} G_{j}(\operatorname{sinc} h \Omega-\Phi) \frac{1}{h \Omega} \cos \left(t_{j}-t_{0}\right) \Omega w_{j}^{1} \\
+ & \frac{h^{2}}{2} \sum_{j=0}^{n} W_{n-j} G_{j}(\operatorname{sinc} h \Omega-\Phi) \frac{1}{h \Omega} \sin \left(t_{j}-t_{0}\right) \Omega w_{j}^{2},
\end{aligned}
$$

where

$$
\begin{aligned}
& w_{j}^{1}:=\Omega y\left(t_{0}\right)+\int_{t_{0}}^{t_{j}} \sin \left(t_{0}-s\right) \Omega \cdot g(y(s)) d s, \\
& w_{j}^{2}:=y^{\prime}\left(t_{0}\right)+\int_{t_{0}}^{t_{j}} \cos \left(t_{0}-s\right) \Omega \cdot g(y(s)) d s .
\end{aligned}
$$

Since

$$
\left\|W_{j+1}-W_{j}\right\| \leq h C \quad \text { and } \quad\left\|w_{j+1}^{1,2}-w_{j}^{1,2}\right\| \leq h C,
$$

partial summation with the sums

$$
\sum_{j=0}^{n}(\operatorname{sinc} \xi-\phi(\xi)) \frac{1}{\xi} \cos j \xi \text { and } \sum_{j=0}^{n}(\operatorname{sinc} \xi-\phi(\xi)) \frac{1}{\xi} \sin j \xi
$$

(due to (14)), shows the bound. (25) is bounded analogously.

Lemma 5. For $n$ with $0 \leq(n+1) h \leq T$, it holds that

$$
\left\|\sum_{j=0}^{n} \Omega \sin (n-j) h \Omega \cdot d_{j}\right\| \leq C h
$$

where $C$ depends on $T, K,\|g\|,\left\|g_{y}\right\|, M_{1}, M_{2}$, and $M_{6}$.

Proof. According to Lemma 1,

$$
\left\|h^{2} \sum_{j=0}^{n} \sin j h \Omega \cdot\left(h \Omega z_{n-j}\right)\right\| \leq h T C
$$

where $C$ depends on $K,\left\|g_{y}\right\|$, and $M_{2}$, we have to bound

$$
\frac{1}{2} h \sum_{j=0}^{n}(h \Omega) \sin j h \Omega \cdot\left(\operatorname{sinc}^{2} \frac{h}{2} \Omega-\Psi\right) g_{n-j}=: \frac{1}{2} h v_{n} .
$$


By partial summation, $v_{n}$ can be written as

$$
v_{n}=E_{n}^{\prime}(h \Omega) g_{0}+\sum_{j=0}^{n-1} E_{j}^{\prime}(h \Omega)\left(g_{n-j}-g_{n-j-1}\right),
$$

where

$$
E_{j}^{\prime}(\xi):=\frac{-\xi}{2 \sin \left(\frac{\xi}{2}\right)}\left(\operatorname{sinc}^{2} \frac{\xi}{2}-\psi(\xi)\right)\left(\cos \left(j \xi+\frac{\xi}{2}\right)-\cos \frac{\xi}{2}\right) .
$$

Due to (15), we have $\left\|E_{j}^{\prime}(h \Omega)\right\| \leq M_{6}$ and therefore

$$
\left\|v_{n}\right\| \leq M_{6}\|g\|+\sum_{j=0}^{n-1} M_{6} M_{1}\left\|g_{y}\right\| h K \leq M_{6}\left(\|g\|+T M_{1}\left\|g_{y}\right\| K\right)
$$

yields the desired result.

Lemma 6. For $n$ with $0 \leq(n+1) h \leq T$, it holds that

$$
\left\|\sum_{j=0}^{n} \cos (n-j) h \Omega \cdot d_{j}^{\prime}\right\| \leq C h
$$

where $C$ depends on $T, K,\|g\|,\left\|g_{y}\right\|,\left\|g_{y y}\right\|, M_{1}, M_{2}$, and $M_{7}$.

Proof. From Lemma 2 we have

$$
\begin{aligned}
\sum_{j=0}^{n} \cos (n-j) h \Omega \cdot\left\{\frac{1}{2} h(\operatorname{sinc} h \Omega\right. & \left.-\Psi_{0}\right) g\left(\Phi y\left(t_{j}\right)\right) \\
& \left.+\frac{1}{2} h\left(\operatorname{sinc} h \Omega-\Psi_{1}\right) g\left(\Phi y\left(t_{j}+h\right)\right)\right\} .
\end{aligned}
$$

This can be bounded by using partial summation.

\section{References}

[1] U. M. Ascher and S. Reich. On some difficulties in integrating highly oscillatory Hamiltonian systems. In Proc. Computational Molecular Dynamics, Springer Lecture Notes, pages 281-296, 1999.

[2] D. Cohen. Conservation properties of numerical integrators for highly oscillatory Hamiltonian systems. Preprint, January, 2005.

[3] D. Cohen, E. Hairer, and Ch. Lubich. Modulated Fourier expansions of highly oscillatory differential equations. Foundations of Comput. Maths., 3:327-450, 2003.

[4] D. Cohen, E. Hairer, and Ch. Lubich. Numerical energy conservation for multi-frequency oscillatory differential equations. BIT, 45:287-305, 2005.

[5] P. Deufhard. A study of extrapolation methods based on multistep schemes without parasitic solutions. Z. angew. Math. Phys., 30:177-189, 1979.

[6] B. García-Archilla, J. Sanz-Serna, and R. Skeel. Long-time-step methods for oscillatory differential equations. SIAM J. Sci. Comput., 30(3):930-963, 1998.

[7] W. Gautschi. Numerical integration of ordinary differential equations based on trigonometric polynomials. Numer. Math., 3:381-397, 1961.

[8] V. Grimm. Exponentielle Integratoren als Lange-Zeitschritt-Verfahren für oszillatorische Differentialgleichungen zweiter Ordnung, PhD thesis, Mathematisches Institut, Universität Düsseldorf, Germany, 2002. 
[9] V. Grimm. A note on the Gautschi-type method for oscillatory second-order differential equations. Numer. Math., 102:61-66, 2005.

[10] V. Grimm. On error bounds for the Gautschi-type exponential integrator applied to oscillatory second-order differential equations. Numer. Math., 100:71-89, 2005.

[11] E. Hairer and C. Lubich. Long-time energy conservation of numerical methods for oscillatory differential equations. SIAM J. Numer. Anal., 38:414-441, 2000.

[12] E. Hairer, Ch. Lubich, and G. Wanner. Geometric Numerical Integration. Springer-Verlag, 2002.

[13] M. Hochbruck and Ch. Lubich. A Gautschi-type method for oscillatory second-order differential equations. Numer. Math., 83:403-426, 1999.

[14] A. Iserles. On the global error of discretization methods for highly-oscillatory ordinary differential equations. BIT, 42:561-599, 2002.

[15] A. Iserles. Think globally, act locally: solving highly-oscillatory ordinary differential equations. Appld. Num. Anal., 43:145-160, 2002.

[16] A. Iserles. On the method of Neumann series for highly oscillatory equations. BIT, 44:473-488, 2004 .

[17] K. Lorenz, T. Jahnke, and Ch. Lubich. Adiabatic integrators for highly oscillatory second order linear differential equations with time-varying eigendecomposition. BIT, 45:91-115, 2005.

[18] L. Petzold, L. Jay, and J. Yen. Numerical Solution of Highly Oscillatory Ordinary Differential Equations. Acta Numerica, 6:437-484, 1997. 